\title{
Technical Notes \\ Free-to-Pivot Flat Plates in Hover for Reynolds Numbers 14 to 21,200
}

\author{
Kenneth O. Granlund* and Michael V. Olı \\ U.S. Air Force Research Laboratory, Wright-Patterson \\ Air Force Base, Ohio 45433-7542 \\ and \\ Luis P. Bernalı \\ University of Michigan, Ann Arbor, Michigan 48109-2140 \\ DOI: $\underline{10.2514 / 1 . J 053169}$
}

\begin{aligned} & \multicolumn{1}{c}{ Nomenclature } \\ $A & =$ motion translation amplitude \\ $b & =$ plate span; $275 \mathrm{~mm} \\ C_{N} & =$ motion-parallel force coefficient $\left(N / 0.5 \rho b c U_{\mathrm{ref}}^{2}\right) \\ C_{P} & =$ motion-parallel power coefficient \\ & $\left(\int_{0}^{1} C_{N}(t / T) U(t / T) / U_{\mathrm{ref}}^{2}\right) \\ C_{T} & =$ motion-normal force (thrust) coefficient \\ $C_{Z} & =$ plate-normal force coefficient \\ $c & =$ plate chord; $50 \mathrm{~mm} \\ f & =$ translation frequency \\ $h_{0} & =$ nondimensional translation amplitude; $A / c \\ R e & =$ Reynolds number based on maximum velocity \\ $S t & =$ Strouhal number $(f c / A 2 \pi f) \\ t / T & =$ phase of plate leading edge imposed motion; \\ $U_{\text {ref }}= & 0 \leq t / T \leq 1\end{aligned}$

\section{Introduction}

$\mathbf{T}$ HE so-called normal hover [1- $\underline{-}$ ] has become a standard problem in the unsteady aerodynamics of periodic oscillation of a flat plate undergoing large excursions in incidence angle. As the motion is a rectilinear analog of a hovering rotor, intuitive questions include the figure of merit. Because of large incidence angles, the problem resembles the classical linear pitch ramp [6]. As there is no imposed relative freestream, the Reynolds number depends on the plate's frequency, amplitude of motion, and kinematic viscosity of the working fluid. This allows for large variations in Reynolds number, assessment of Reynolds number effects on vortex formation and shedding, thrust production, and propulsive efficiency. Here, we extend our work on rigid flat plates hinged at the leading edge and oscillated sinusoidally in a water tank [7], to the same motion in glycerin/water mixtures, enabling Reynolds number variations based on plate chord and leading-edge maximum speeds from $O(10)$ to

Received 30 October 2013; revision received 11 February 2014; accepted for publication 25 February 2014; published online 4 June 2014. This material is declared a work of the U.S. Government and is not subject to copyright protection in the United States. Copies of this paper may be made for personal or internal use, on condition that the copier pay the $\$ 10.00$ per-copy fee to the Copyright Clearance Center, Inc., 222 Rosewood Drive, Danvers, MA 01923; include the code 1533-385X/14 and \$10.00 in correspondence with the CCC.

*Aerospace Systems Directorate; kenneth.granlund@wpafb.af.mil. Senior Member AIAA.

${ }^{\dagger}$ Aerospace Systems Directorate. Associate Fellow AIAA.

\#Department of Aerospace Engineering. Senior Member AIAA.
$O(10,000)$ to cover the $R e=200$ computations of Wan et al. [4] and Gaston et al. [].

The kinematics are motivated by a flight control scheme proposed by Doman et al. [9] for the flapping-wing configuration developed by Wood [10], where the wing leading edge is directly actuated in a sweeping motion, but the wing incidence angle is free to float between limiters. The incidence angle is at the limiter (45 deg) throughout the translation phase of each semistroke, with a rotation from one limiter side to the other, at or near the extrema of each semistroke.

\section{Experimental Setup}

The U.S. Air Force Research Laboratory's Horizontal FreeSurface Water Tunnel is fitted with a three-degree-of-freedom electric rig [11], here used only in fore-aft translation of the test article. Motion is controlled via a Galil DMC-4040 motion controller from preprogrammed scripts achieving less than $0.1 \mathrm{~mm}$ linear and less than $0.2 \mathrm{deg}$ angular position errors. A $6 \%$ thick carbon-fiber flat plate with square edges (and an aspect ratio of 5.5) is hinged at the midspan of its leading edge and constrained to rotate within $\pm 45^{\circ}$ from its rest (vertical) position by mechanical stops in the strut holding the leading-edge pivot, shown in Fig. 1. As the support is translated, the pressure difference between the advancing and retreating sides of the plate forces the plate toward one of its pitch limiters. This is reversed in the opposite direction of translation. The test section floor is $\approx 6$ chords below the plate's trailing edge, which minimizes the influence of ground effect [12]. The test section walls are $\approx 1.5$ chords away from the model. The aspect ratio effect is small, as noted by Granlund et al. [7]. To vary the Reynolds number over a larger range than possible with just the velocity and size of the model, the working fluid is a mixture between $100 \%$ glycerin and $100 \%$ water, giving a chord-based Reynolds number range of 14 to 21,200 , respectively, at $25^{\circ} \mathrm{C}$ [13]. The experiment is in a 2.4-m-long 0.6-mhigh 0.45-m-wide Plexiglas tank placed inside the tunnel test section, with the rest of the tunnel (that is, outside of the tank) filled with water.

Force measurements were done with an ATI Nano25 IP68 sixcomponent balance, sampled at $1 \mathrm{kHz}$ and low-pass filtered at $34 \mathrm{~Hz}$ in hardware, and then sampled at 50 times of the motion frequency using a fourth-order Chebychev II low-pass filter in MATLAB. All motions are repeated for 20 cycles, with the first three not included in averaging. Force data are nondimensionalized based on plate area, density of the fluid mixture, and either instantaneous or maximum leading-edge translation velocities. Time is nondimensionalized by motion period. The thrust coefficient $C_{T}$ is in the direction normal to the fore-aft motion of the plate's leading edge, whereas the motionparallel force coefficient $C_{N}$ is parallel to the leading-edge motion, as depicted in Fig. 1. Uncertainty in the force coefficients is largest during the force "spike" but less than 0.1 during the translation part of the motion.

\section{Results}

The thrust coefficient, motion-parallel coefficient, and platenormal coefficients are shown in Fig. 2, for Strouhal numbers from 0.025 through 0.2 and Reynolds numbers from 28 through 21,200, based on plate chord and leading-edge translational speed. Strouhal number for hover, in the absence of a freestream, is based on kinematics [14,15]. Thrust and motion-parallel coefficients are normalized by semistroke maximum speed, whereas the coefficient of force normal to the plate is normalized by the instantaneous leading-edge translation speed, resulting in a division by zero at the semistroke extrema $(t / T=0,0.5,1.0$, etc.). This approach is useful 


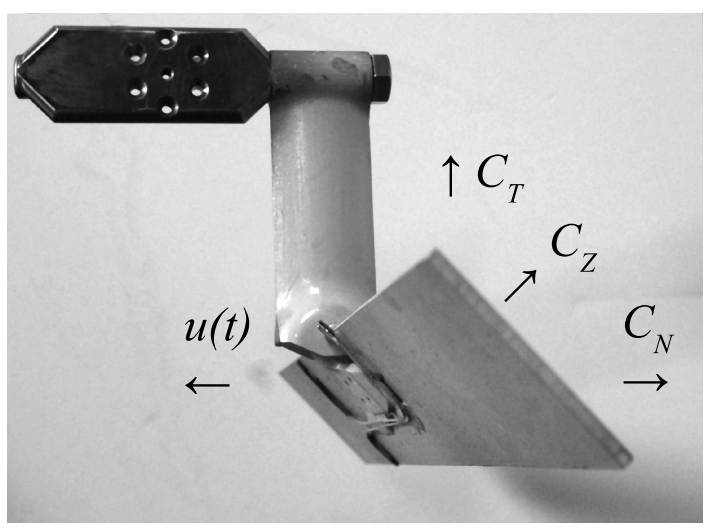

Fig. 1 Free-to-pivot plate with motion and force coefficients defined. for assessment of the extent to which the aerodynamic response during the translational portion of each semistroke is quasi steady. That is, because the plate incidence angle is constant, a true quasisteady response would mean an identically constant value of force, under this normalization. According to Sane and Dickinson [2], $C_{Z}=2.5$ for constant translation at $45 \mathrm{deg}$ at $R e=140$. One sees that, for all Reynolds numbers at Strouhal numbers of 0.025 and 0.05 , the force coefficient declines nearly linearly from this value with ascending $t / T$ values during the translational portion of the semistroke. For a Strouhal number of 0.1 , this quasi-steady assumption is not unreasonable for the majority of the translation part of the motion.

The $S t=0.05$ and higher Strouhal number cases in Fig. 2 are characterized by a spike in $C_{T}$ and $C_{N}$, which increases in relative magnitude with increasing $S t$ and is associable with the plate completing its rotation near each semistroke extremum and striking
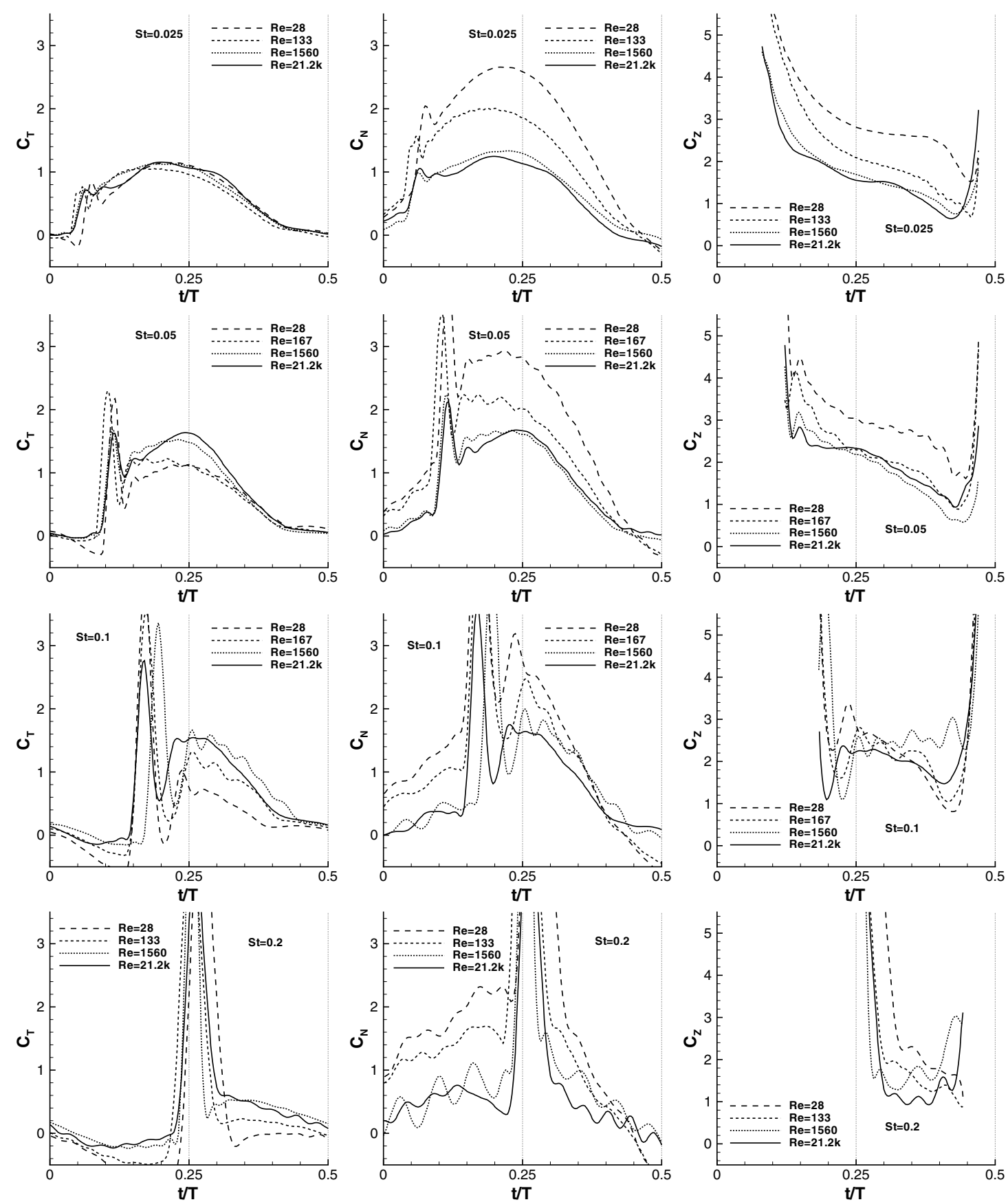

Fig. 2 Thrust (left), motion-parallel (middle), and plate-normal (right column) coefficients for translation at $28<R e<21,200$. Rows are from top to bottom: $S t=0.025,0.05,0.1$, and 0.2 . 


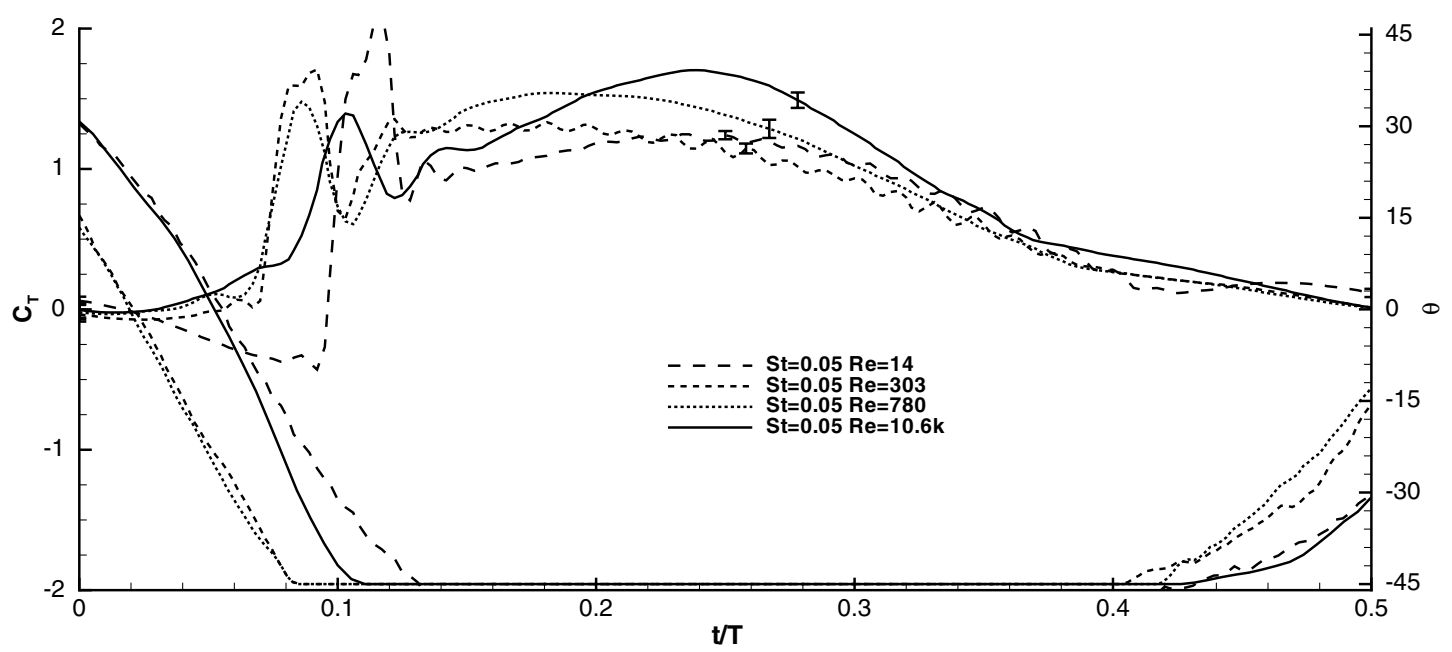

Fig. 3 Superposition of incidence angle and thrust coefficient with uncertainty bounds for $S t=0.05$ and $14<R e<10,600$ over one semistroke of plate leading-edge translation.

its incidence limiter [7]. The motion-parallel coefficient required to drive the plate leading edge is higher for a lower Reynolds number, but it relaxes to weak Reynolds number dependency above $R e=$ $O\left(10^{3}\right)$. Bos et al. [16] also came to the same conclusion for a highRossby-number flapping elliptic wing. On the other hand, the thrust coefficient does not display a monotonic trend, as it is nearly Reynolds number independent for $S t=0.025$ but becomes progressively lower at lower Reynolds number for higher Strouhal numbers. The coefficient of force normal to the plate, normalized on instantaneous motion velocity, is higher at lower Reynolds numbers for $S t=0.025$ and $S t=0.05$, but dependency on Reynolds number becomes less clear at higher Strouhal numbers.

Plate kinematics (that is, the phase during each semistroke that rotation occurs) is not completely independent of Reynolds number (Fig. 3, St $=0.05$ ), but the dependency is weak. For the intermediate Reynolds numbers, the plate has slightly less phase delay than for $R e=14$ and $\operatorname{Re}=10,600$. That is, a phase-neutral motion (normal hover) would have the plate at a zero pitch angle, precisely at every semistroke extremum. History of the plate-normal force coefficient in Fig. 3 shows that force spikes coincide in $t / T$ with the point where the plate strikes against its incidence limiter. In other words, the force spike is not centered in $t / T$ about the midpoint of the plate's rotational motion, and it is therefore not due to the rotation itself [7].
Further, Reynolds number dependency of forces correlates closely with how the rotational kinematics depends on the Reynolds number.

From the standpoint of hovering flapping-wing flight-article performance, stroke-averaged thrust- and motion-parallel force coefficients are more important than their instantaneous values. One is thus concerned with dependency of these averaged coefficients on Strouhal number and Reynolds number: the latter being a proxy for flight-article size. Hover efficiency, reported as a figure of merit [7], combines $C_{T}$ and $C_{N}$, with the latter multiplied by leading-edge translation speed to form a power coefficient $C_{P}$ [FOM; ideal-toactual thrust power in hover; $\left.\overline{C_{T}}{ }^{2 / 3} / \overline{C_{P}}\left(8 h_{0}\right)^{1 / 2}\right]$. Figure $\underline{4}$ shows that the figure of merit increases monotonically with increasing Reynolds numbers from $O(10)$ to $O(10,000)$. The optimal Strouhal number for hover (that is, the highest figure of merit) is between 0.05 and 0.1, with a shift to higher Strouhal numbers at higher Reynolds number. Noting from Fig. 2 that the Reynolds number dependency of the thrust coefficient is lower than that of the motion-parallel force coefficient, the increase in figure of merit with increasing Reynolds numbers ought to intuitively be imputed primarily to decrease in viscous resistance.

The stroke-averaged thrust coefficient reaches a maximum of $C_{T} \approx 0.7$ for $\operatorname{Re}>10^{3}$ (Fig. 5). Maximum $C_{T}$ is generated at $S t \approx 0.05$, irrespective of Reynolds number. Thus, the Strouhal
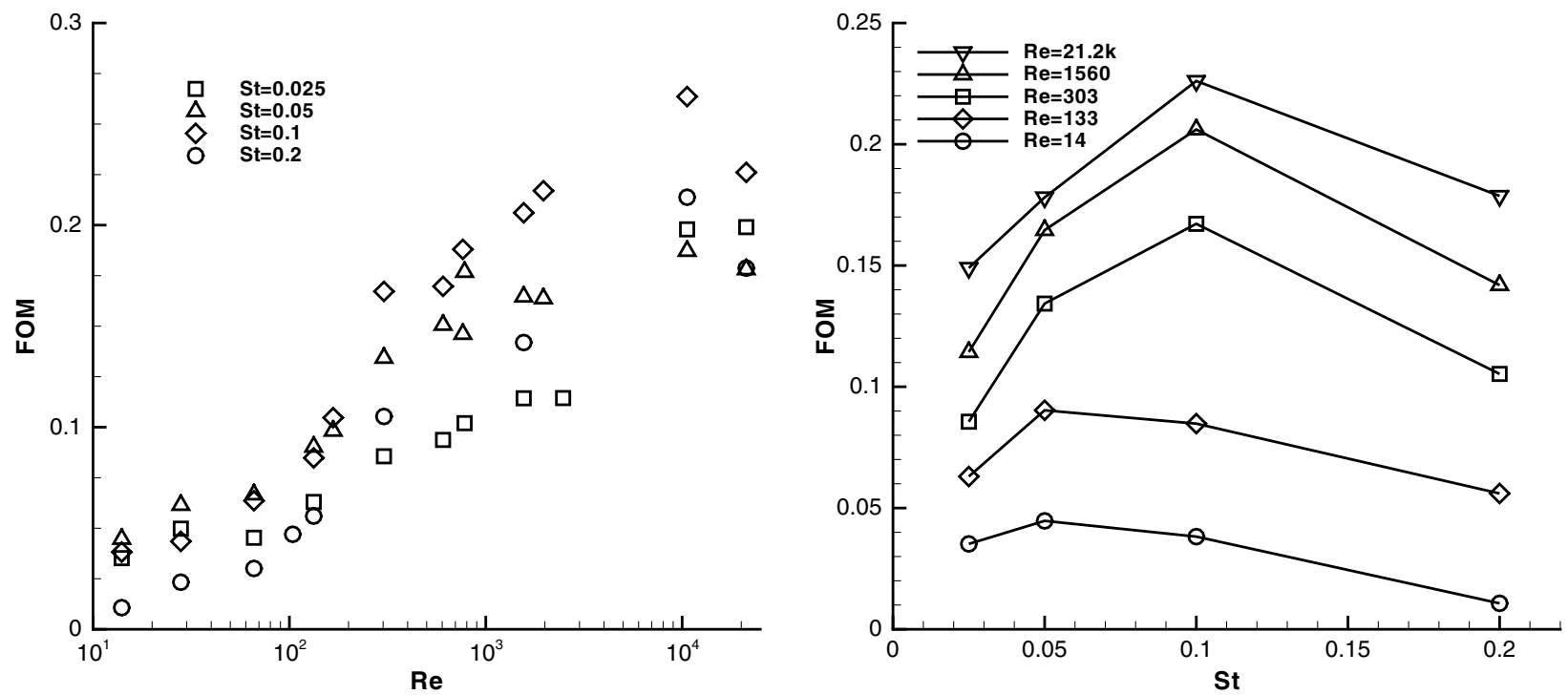

Fig. 4 Figure of merit as a function of Reynolds number and Strouhal number: FOM vs Reynolds number for various Strouhal numbers (left); and complementary presentation, FOM vs Strouhal number for various Reynolds numbers (right). 

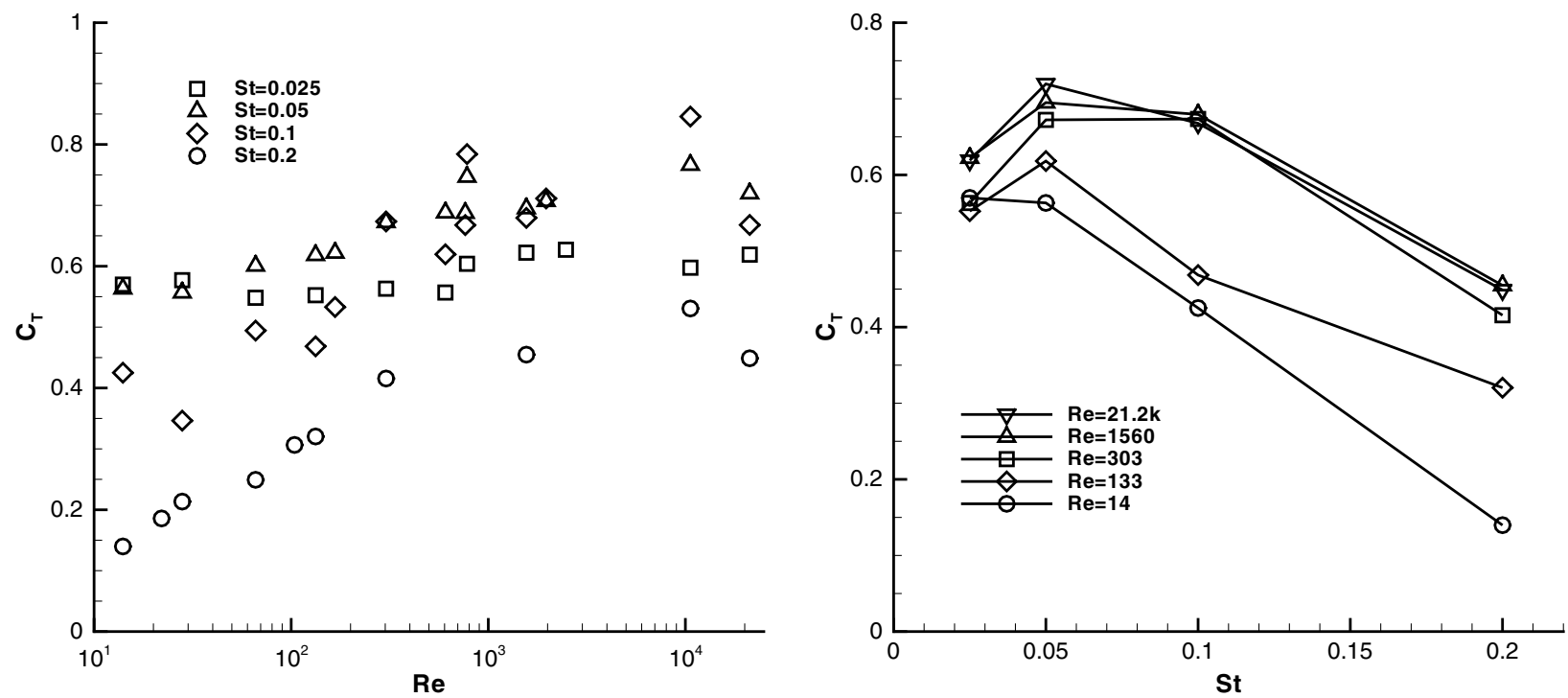

Fig. 5 Stroke-averaged thrust coefficient as a function of Reynolds number and Strouhal number: $C_{T}$ vs Reynolds number for various Strouhal numbers (left); and complementary presentation, $C_{T}$ vs Strouhal number for various Reynolds numbers (right).

number for the highest figure of merit occurs at somewhat lower Strouhal numbers than for a maximum stroke-averaged thrust coefficient. For $S t>0.05$, the stroke-averaged thrust coefficient is reduced dramatically when the Reynolds number is reduced. Part of the explanation for the reduced stroke-averaged thrust coefficient for the lower-Reynolds-number and higher-Strouhal-number cases is evident from the negative instantaneous thrust-coefficient values for said cases visible in Fig. 2 for $t / T$ preceding the spike in thrust coefficient. Thus, the inefficiency of hover at high Strouhal numbers (that is, the small ratio of stroke amplitude to wing chord) is exacerbated at lower Reynolds numbers, both in terms of reduced thrust available and lower figure of merit.

\section{Conclusions}

The stroke-averaged thrust and efficiency of a free-to-pivot rectangular translating plate, as a function of amplitude and Reynolds number, are investigated by direct force measurements in a tank filled with a mixture of glycerin and water. Across four orders of magnitude of Reynolds numbers, maximum stroke-averaged thrust is produced at $S t \approx 0.05$. Peak efficiency in hover is produced $0.05<S t<0.1$, with a monotonic increase for higher Reynolds numbers.

\section{References}

[1] Freymuth, P., "Thrust Generation by an Airfoil in Hover Modes," Experiments in Fluids, Vol. 9, Nos. 1-2, 1990, pp. 17-24. doi:10.1007/BF00575331

[2] Sane, S. P., and Dickinson, M. H., "The Control of Flight Force by a Flapping Wing: Lift and Drag Production," Journal of Experimental Biology, Vol. 204, Aug. 2001, pp. 2607-2626.

[3] Dickson, W., and Dickinson, M., "The Effect of Advance Ratio on the Aerodynamics of Revolving Wings," Journal of Experimental Biology, Vol. 207, Nov. 2004, pp. 4269-4281. doi: $10.1242 /$ jeb.01266

[4] Wan, H., Dong, H., and Huang, G., "Hovering Hinge-Connected Flapping Plate with Passive Deflection," AIAA Journal, Vol. 50, No. 9 , 2012, pp. 2020-2026. doi:10.2514/1.J051375

[5] Shyy, W., Aono, H., Kang, C.-k., and Liu, H., An Introduction to Flapping Wing Aerodynamics, Cambridge Univ. Press, New York, 2013, pp. 95-116.
[6] Granlund, K., Ol, M., and Bernal, L., "Unsteady Pitching Flat Plates," Journal of Fluid Mechanics, Vol. 733, Oct. 2013, p. R5. doi: $10.1017 / \mathrm{jfm} .2013 .444$

[7] Granlund, K., Ol, M., and Bernal, L., "Quasi-Steady Response of Freeto-Pivot Flat Plates in Hover," Journal of Fluids and Structures, Vol. 40, July 2013, pp. 337-355. doi:10.1016/j.jfluidstructs.2013.02.020

[8] Gaston, Z., Wan, H., Dong, H., and Ol, M., "Analysis of a HingeConnected Flapping Plate with an Implemented Torsional Spring Model," AIAA Paper 2012-0298, 2012.

[9] Doman, D., Oppenheimer, M., and Sigthorsson, D., "Wingbeat Shape Modulation for Flapping-Wing Micro-Air-Vehicle Control During Hover," Journal of Guidance, Control, and Dynamics, Vol. 33, No. 3, 2010, pp. 724-739. doi: $10.2514 / 1.47146$

[10] Wood, R., "The First Takeoff of a Biologically Inspired At-Scale Robotic Insect," IEEE Transactions on Robotics, Vol. 24, No. 2, 2007, pp. 341-347. doi:10.1109/TRO.2008.916997

[11] Ol, M. V., Bernal, L. P., Kang, C.-K., and Shyy, W., "Shallow and Deep Dynamic Stall for Flapping Low Reynolds Number Airfoils," Experiments in Fluids, Vol. 46, No. 5, 2009, pp. 883-901. doi:10.1007/s00348-009-0660-3

[12] Chabalko, C., Fitzgerald, T., Valdez, M., and Balachandran, B., "Flapping Aerodynamics and Ground Effect," AIAA Paper 2012-0420, 2012.

[13] Minier, C. S., and Dalton, N. N., Physical Properties of Glycerine and it's Solutions, American Chemical Society Monograph 117, Reinhold, New York, 1953, p. 10.

[14] Poelma, C., Dickson, W., and Dickinson, M. H., "Time-Resolved Reconstruction of the Full Velocity Field Around a Dynamically-Scaled Flapping Wing," Experiments in Fluids, Vol. 41, No. 2, 2006, pp. 213 225. doi:10.1007/s00348-006-0172-3

[15] Dong, H., Lian, Z., and Harff, M., "Optimal Settings of Aerodynamic Performance Parameters in Hovering Flight," International Journal of Micro Air Vehicles, Vol. 1, No. 3, Sept. 2009, pp. 173-181. doi:10.1260/175682909789996195

[16] Bos, F. M., van Oudheusden, B., and Bijl, H., "Wing Performance and 3D Vortical Structure Formation in Flapping Flight," Journal of Fluids and Structures, Vol. 42, Oct. 2013, pp. 130-151. doi:10.1016/j.jfluidstructs.2013.04.002

I. Gursul Associate Editor 